\title{
Open Circuit
}

National Cancer Institute

\section{Source}

National Cancer Institute. Open Circuit. NCI Thesaurus. Code C92087.

Problem due to an electrical circuit that does not conduct current because a switch is open, a wire is broken, etc. 